\title{
Tuning of the Tectorial Membrane in the Basilar Papilla of the Northern Leopard Frog
}

\author{
R. L. M. Schoffelen, ${ }^{1,2}$ J. M. Segenhout, ${ }^{1}$ and P. van DijK ${ }^{1,2}$ \\ ${ }^{1}$ Department of Otorhinolaryngology/Head and Neck Surgery, University Medical Center Groningen, P.O. Box 30001, \\ 9700RB, Groningen, The Netherlands \\ ${ }^{2}$ Faculty of Medical Sciences, School of Behavioral and Cognitive Neurosciences, University of Groningen, P.O.Box 196, \\ 9700AD, Groningen, The Netherlands
}

Received: 9 January 2009; Accepted: 9 March 2009; Online publication: 2 June 2009

\begin{abstract}
The basilar papilla (BP) in the frog inner ear is a relatively simple auditory receptor. Its hair cells are embedded in a stiff support structure, with the stereovilli connecting to a flexible tectorial membrane (TM). Acoustic energy passing the papilla presumably causes displacement of the TM, which in turn deflects the stereovilli and stimulates the hair cells. Auditory neurons that contact the BP's hair cells are known to have nearly identical characteristic frequencies and frequency selectivity. In this paper, we present optical measurements of the mechanical response of the TM. Results were obtained from five specimens. The TM displacement was essentially in phase across the membrane, with the largest amplitudes occurring near the hair cells. The response was tuned to a frequency near $2 \mathrm{kHz}$. The phase accumulated over at least $270^{\circ}$ across the measured frequencies. The tuning quality $\mathrm{Q}_{10 d B}$ values were calculated; the average $\mathrm{Q}_{10 d B}$ was $2.0 \pm 0.8$ (standard deviation). Our results are comparable to those of neural-tuning curves in the same and a similar species. Also, they are in agreement with the response of an associated structure - the contact membrane-in a closely related species. Our data provides evidence for a mechanical basis for the frequency selectivity of the frog's BP.
\end{abstract}

Correspondence to: P. van Dijk · Department of Otorhinolaryngology/ Head and Neck Surgery - University Medical Center Groningen . P.O. Box 30001, 9700RB, Groningen, The Netherlands. Telephone: +31-50-3612550; fax: +31-50-3611698; email: p.van.dijk@med.umcg.nl
Keywords: amphibian, anuran, anuran ear, frog ear, mechanical tuning

\section{INTRODUCTION}

The anuran inner ear provides us with an opportunity to study a variation of the vertebrate auditory receptor that is a lot less complex than the mammalian cochlea. The anatomy of the frog inner ear (Fig. 1) differs significantly from that of the mammalian inner ear. It contains two auditory end organs, the amphibian papilla (AP) and the basilar papilla. Both the AP and the BP have hair cells as their mechano-electrical transducers. However, they lack a basilar membrane as the flexible substrate for these cells. Instead, the hair cells are set in limbic tissue (Wever 1985), with the stereovilli protruding into the endolymphatic space and connecting to the overlying TM.

The AP is the frog's low-frequency detector. It is the more elaborate and complex of the two auditory end organs; it contains more hair cells, with varying orientations; it has a more elaborate TM; and it is - partiallyefferently innervated (Simmons et al. 2007). Functionally, the AP exhibits tonotopy (Lewis et al. 1982), with neural characteristic frequencies from approximately 100 up to $1,400 \mathrm{~Hz}$; with the upper limit depending on the species (reviewed in Ronken 1991). Neural tuning sharpness in the AP is similar to that of other vertebrate auditory organs in the same frequency range (Evans 1975). Also, spontaneous otoacoustic emissions are 
FIG. 1. Frog ear anatomy and orientation in the measurement setup. A Schematic cross-section of the frog ear (adapted from Wever 1985). On the right, the air-filled middle ear is displayed with the tympanic membrane and the columella. On the left, the fluid-filled inner ear is shown, with the perilymphatic fluid in white and the endolymphatic fluid in gray. The tectorial membranes of both the AP and the BP are indicated in red. The oval window is covered by the footplate $(F)$ of the columella and the operculum $(O p)$. B Schematic of the preparation and its orientation in the experimental setup. The preparation is rotated approximately $90^{\circ}$ relative to panel $\mathbf{A}$. The arrow indicates the microscope's viewing direction; the green dashed line indicates the line of sight. The stimulator $(S)$, placed against the operculum, is indicated in blue on the left. The dashed gray outline indicates the area detailed in panel C. C Cross-section of the frog's BP anatomy (based on Frishkopf and Flock 1974; R. catesbeiana). The arrow again indicates the viewing direction. Labels: AP amphibian papilla, BP basilar papilla, CM contact membrane (purple), $E$ endolymph, Ep epithelium (blue), $F$ columella footplate, $M M$ middle-ear muscles, $N$ nerve fibers (yellow), $O$ microscope objective, $O p$ operculum, $P$ perilymph, $S$ stimulator (blue), Sacc sacculus, $T M$ tectorial membrane (red).

present (Van Dijk et al. 1996; Van Dijk and Meenderink 2006) and distortion product otoacoustic emissions are physiologically vulnerable (Van Dijk et al. 2003) in the frequency range of the AP.

The BP (Fig. 1C) covers the higher portion of the frog's auditory frequency range. It is a small organ, with only about 60 hair cells in the bullfrog (Frishkopf and Flock 1974), and about 75 in the northern leopard frog (personal observation, JM Segenhout and RLM Schoffelen). It has no known electrical tuning and the presence of efferent innervation may be species dependent (Robbins et al. 1967; Frishkopf and Flock 1974). Afferent nerve fibers connecting to the BP have very similar tuning curves within an individual animal, and the sharpness of tuning is lower than that observed in other tectorial hearing organs in the same frequency range (Ronken 1990; Evans 1975). In other words, the BP is broadly tuned to a single characteristic frequency. This frequency may vary between individuals of a particular species, and ranges from about 1.2 to $4 \mathrm{kHz}$ across species studied (reviewed Ronken 1991; Schoffelen et al. 2008).

In contrast with the AP's frequency range, no spontaneous otoacoustic emissions can be recorded in the BP's range (Van Dijk et al. 1996). In addition to that, distortion-product otoacoustic emissions in the BP's range are less vulnerable to physiological insults than those in the AP's range (Van Dijk et al. 2003). Recently, Meenderink et al. (2005a) showed that both the amplitude and phase characteristics of the BP's distortion-product otoacoustic emissions can bequalitatively-explained by assuming that the BP functions as a single passive nonlinear auditory filter.

In the current study, we focus on the response of the TM in the frog's BP. Purgue and Narins (2000a) measured the response of the contact membrane of both the papillae. In case of the BP, the contact

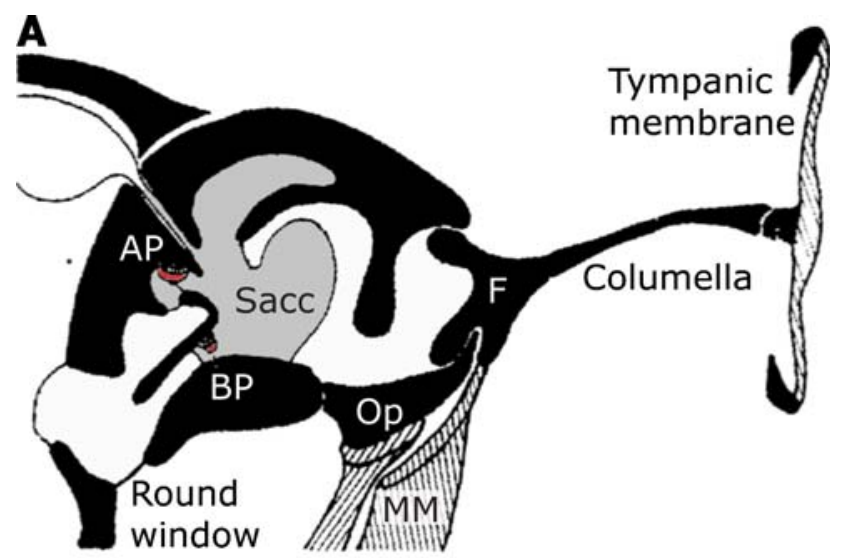

B

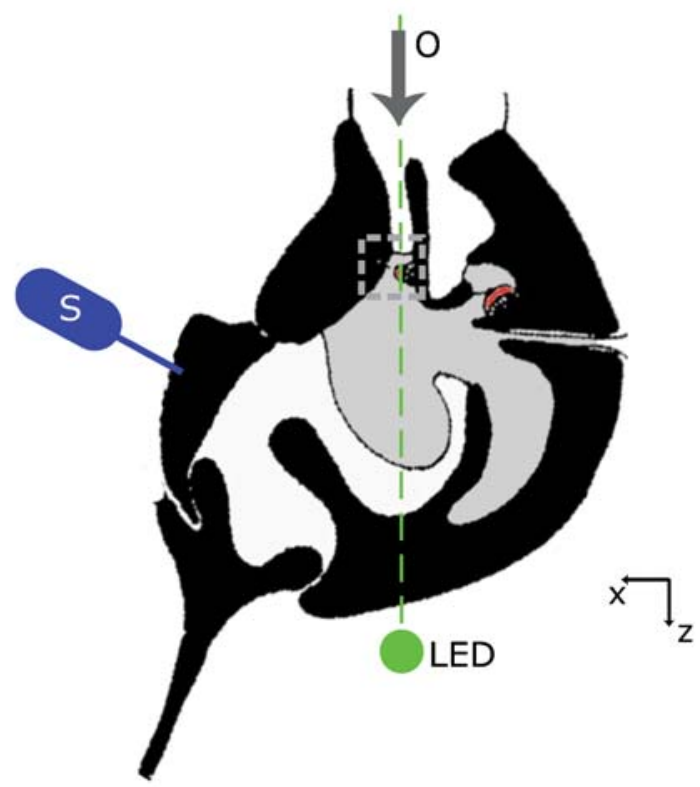

C

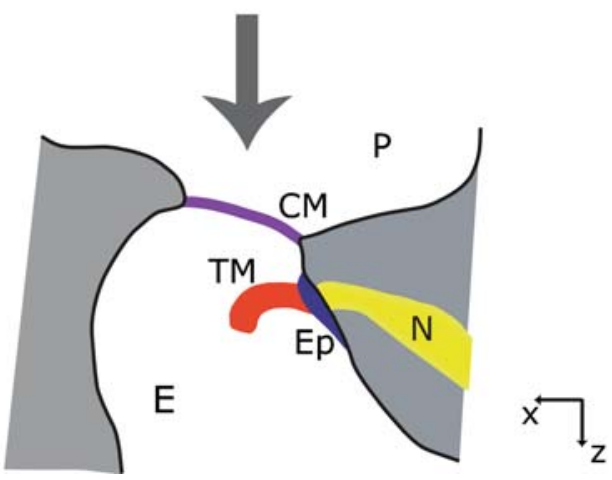

membrane is close to the sensory epithelium and separates the endolymphatic space from the perilymphatic space (Fig. 1C). Its response is tuned to a frequency close to the typical characteristic frequencies of afferents connecting to the BP. Based on this correspondence, we hypothesize that the tuning of the $\mathrm{BP}$ must be mechanical in nature. Therefore, the tuning should be observable in the response of the TM. As will be shown in our results, the mechanical response of the 
TM can indeed account for the frequency selectivity as observed in the auditory neurons.

\section{MATERIALS AND METHODS}

Animals and surgical procedures

Northern leopard frogs, Rana pipiens pipiens, were used in all experiments; data presented here was obtained from five animals $(71 \pm 29 \mathrm{~g}$; one male, four females). The animals were obtained from a commercial supplier (Charles D. Sullivan Co. Inc., Nashville (TN), USA via Exoterra Schaudi GmbH, Holzheim, Germany) and housed at the University of Groningen laboratory-animal facilities. All the experiments described were conducted in compliance with the current legislation on animal experiments in The Netherlands. Part of these requirements is the approval of the experimental procedure by the Institutional Animal Care and Use Committee prior to the start of the measurements.

The animal was killed using a double-pith procedure at the start of the experiment. The posterior part of the skull, containing the ears, was isolated and split sagitally. This resulted in two preparations, each containing an ear. Each of the preparations was kept submerged in amphibian ringer solution (Carolina Biological Supply Company, Burlington, NC, USA) during all further procedures. One preparation was chosen for immediate further treatment and subsequent experimentation. The other preparation was stored in a refrigerator, at approximately $5^{\circ} \mathrm{C}$. It remained there until approximately an hour before the experiments on the first preparation would end. At that time, it was brought out and underwent the same treatment as the first preparation. The storage at low temperature did not appear to affect the specimen to any degree observable by inspection under the microscope or in the outcome of our measurements. Therefore, the distinction between the first and the second preparation is not explicitly made from this point on.

The tympanic membrane and the lateral part of the columella were removed. Any soft tissue and most of the bone were removed, leaving part of the skull in place for clamping the preparation. The perilymphatic space was opened at the round window to allow a direct view into the basilar papilla's lumen through its contact membrane. The dissection procedure allowed for the endolymphatic space to be kept intact, except for the duct to the endolympatic sac in the brain cavity. In a control experiment, the contact membrane of the basilar papilla was punctured. The resulting mixing of endolymph fluid and ringer solution led to visible degeneration of hair cells, as observed by somatic swelling. Such swelling was never observed in the regular experiments, suggesting that the endolymph fluid is well separated from the surrounding ringer solution.

Finally, a perspex cylindrical prosthesis was glued onto the operculum to facilitate the application of the stimulus device (see below).

\section{Measurement setup}

The heart of the measurement setup was a trifocal microscope (Olympus Corporation, Japan) which sat on a vibration-isolation table inside a sound-isolating booth. Any equipment that was not mounted directly on the microscope was kept outside of the booth, except for the stimulator's amplifier (see below). The sound-isolating booth was darkened to avoid scattered light from being picked up by the camera.

The microscope had $5 \times(\mathrm{NA}=0.10)$ and $40 \times(\mathrm{NA}=$ $0.80)$ objectives. The focal plane of the $40 \times$ objective could be controlled with a piezo-electric positioner (Physik Instrumente GmbH and Co., Karlsruhe, Germany). The microscope's lighting system was adapted to house a green Luxeon power LED (Lumileds Lighting, San Jose, CA, USA). A scientific grade digital camera (DVC Company, Austin, TX, USA) was mounted on the microscope with $0.5 \times$ video adapter. Its sensor was a $1,392 \times 1,040$ pixels $(8.9 \times 6.7 \mathrm{~mm})$ monochrome $\mathrm{CCD}$ chip with 12-bit pixel depth.

The stimulation device was a closed-loop piezo actuator (Physik Instrumente). The actuator was placed in a micro-positioner (Kanetec, Co., Ltd., Kyoto, Japan) sitting on the microscope table. A needle on the piezo stack was used to connect it to the preparation's operculum prosthesis.

An industrial-grade computer (National Instruments, Austin, TX, USA) was used to automate the experiment. Signal control, image acquisition, and data storage were programmed in LabView v8.2 (National Instruments). A camera-interface card and a high-frequency I/O card (both National instruments) were used to connect to the measurement hardware. A signal generator (Stanford Research Systems Inc., Sunnyvale, CA, USA) triggered the start of stimulation and illumination signals. The feedback of the piezo actuator was read out using an analogue oscilloscope (Hameg Instruments $\mathrm{GmbH}$, Mainhausen, Germany).

\section{Measurement procedure}

The preparation was placed under the microscope and clamped firmly into place (Fig. 1B). The needle of the stimulator was aligned with the operculum's prosthesis. The needle's tip was initially placed closely to the prosthesis. The application of the probe to the prosthesis was done under the $5 \times$ objective. Using the 
micro-positioner, it was moved against the cupped center of the probe; forward motion was stopped at the first observation of movement of the operculum.

Next, the specimen was positioned to optimize the view of the BP's TM under the $40 \times$ objective. The camera was rotated to orientate the edge of the TM vertically in the image. Illumination time and LED voltage were adapted to optimize the use of the camera's pixel depth. The objective's positioner was used to determine the range of focal planes to be used, based on the sharpness of the tectorial structures in the image.

The stimulator displaced the operculum with a sine-wave signal. Stimulation frequencies ranged from 0.5 to $3.0 \mathrm{kHz}$ and were presented in a random order. A 100-ms sinusoidal ramp up and ramp down was used for switching the signal on and off, respectively. The duration of the stimulation signal was determined by the required illumination time to fill the CCD to a sufficient degree $50 \%$ to $75 \%$ of the full range); typically between 1.5 and $3 \mathrm{~s}$. The average stimulus amplitudes used for the various preparations ranged from -23 to $-29 \mathrm{~dB}$ (all reported decibel values are referenced to $1 \mu \mathrm{m}$ ).

The dynamic displacement of the piezo-electrical stimulator was frequency dependent. By adapting the driving voltage, the stimulation amplitude was kept constant within $2.5 \mathrm{~dB}$. In data analysis, the TM response amplitudes were corrected linearly for variations of the stimulus amplitude.

The LED stroboscopically illuminated the specimen with a $10 \%$ duty cycle. No illumination took place during ramp up and ramp down of the stimulus. Images were taken for eight values of equidistantly spaced phases relative to the stimulus signal. The camera was activated throughout the duration of the stimulation. After deactivation, the camera's CCD was read out and stored.

This procedure was repeated for at least 30 focal planes spaced $1 \mu \mathrm{m}$ apart. Each measurement sequence started on a focal plane at the round-window side of the $\mathrm{BP}$ and progressed towards planes on the saccular side. The resulting data consisted of at least 240 images per measurement sequence (stimulus frequency; 30 planes $\times$ 8 phases). These images combined to produce a 3D movie of the TM during one cycle of the stimulation signal. We limited the total measurement time on each preparation to a maximum of $4 \mathrm{~h}$.

An example of an individual measurement image is given in Figure 2A, with the important anatomical features indicated in Figure 2B. The TM occludes approximately half of the BP's lumen. It has a free edge (at the top in Fig. 2), and an approximately semicircular area, when viewed from the roundwindow side. The free edge is firmly attached on both sides to the lumen boundary, and thick compared to
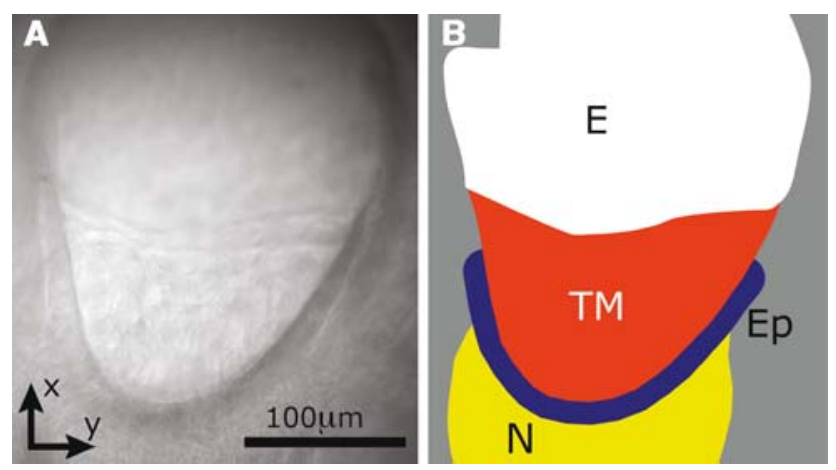

FIG. 2. The basilar papilla of the northern leopard frog as seen from the round window. A Light microscopy image with definition of the $x$ and $y$ directions; the $z$ direction points into the image plane. B Indication of important structures in panel A. E endolymphatic space (white), Ep epithelium (b/ue), $N$ nerve fibers (yellow), TM tympanic membrane (red).

the rest of the TM (personal observation from scanning electron microscopy). The TM thickness, i.e., the extent along the viewing direction ( $z$ direction, Fig. 2), is approximately $50 \mu \mathrm{m}$ (Schoffelen et al. 2007). The sensory epithelium is located under the curved perimeter of the TM (Fig. 2B).

\section{Data analysis}

Preprocessing Preprocessing and motion detection were performed on a dedicated Debian-linux system, using the $\mathrm{nD}$ image analysis package for motion detection (Research Laboratory of Electronics, Massachusetts Institute of Technology, USA; Davis and Freeman 1988). The recorded binary streams were transferred to the $\mathrm{nD}$ file format using custom additions to the package. The images were scaled to have a constant average gray value across recorded phases and planes. The correction factor was generally less than $10 \%$. All further image analysis steps were performed on these scaled versions of the images.

The data was analyzed using two separate procedures, which are described below. Both procedures use the nD's optical-flow algorithms for the estimation of the displacement values. Optical-flow algorithms were originally used for robot vision (Horn and Weldon 1988). They work on the assumption that the intensity of features remains constant between two recorded images (Davis and Freeman 1988). Any variations in intensity between images are then the result of the motion of features relative to the camera. The motion of features can be estimated by taking the two recorded images, defining a region of interest (ROI) to trace in one of them, and using a linear least-squares fit to calculate the displacement that minimizes the difference between the images. 
Conventions The most effective means of stimulating a hair cell is to deflect its hair bundle towards its longest stereovillus (e.g., Lewis 1976). This direction is called the hair-cell orientation. If we approximate the surface area of the hair cell covered by stereovilli with a circle, the hair cell orientation is the direction along a diameter of that circle towards the longest stereovillum. The hair-bundle orientation in the BP of ranid frogs is uniform and always from the sacculus towards the round window (Lewis and Narins 1999; personal observation, JM Segenhout and RLM Schoffelen). We expected the major component of any sound-induced displacement of the TM to be in the direction of the hair-bundle orientation, i.e., to and from the sacculus. This expectation was based on the hair-cell orientation in the BP on one hand, and the anatomy and expected fluid displacement on the other hand (Purgue and Narins 2000a, b).

Our measurement setup allowed us to look into the BP's lumen and onto the TM from the round-window side of the lumen (see Fig. 1). We defined the direction along the line of sight to be the $z$ direction. The $y$ direction was the horizontal direction in the image displayed in Figure 2, and the $x$ direction was the vertical direction in the same image. As a result of the positioning of the specimen and the camera, the $y$ direction was parallel to the free edge of the TM and the $x$ direction was by definition perpendicular to it and in the image plane. The $z$ direction was perpendicular to the image plane.
In Figure 3, a highly schematic view of the BP's lumen, and its TM, was drawn with an indication of the $x z$ and $y z$ cross-sections used below. The hairbundle orientation was presumed to be predominantly in the negative $z$ direction, with the $x$ and $y$ directions perpendicular to it.

Cross-sectional views In order to get an overview of the motion of the TM in the basilar papilla, we used the nD's built-in voxel-analysis functions. The 3D analysis method did not work effectively on our dataset, presumably due to insufficient contrast and sharpness in parts of the images. Therefore, we analyzed the motion in planes oriented in the $x z$ and the $y z$ directions (see Fig. 3A). Within these planes, we obtained displacement estimates for the two orthogonal directions within the plane. An overview of the motion in three dimensions was created by combining the estimates in the perpendicular planes. This method gave us the displacements over time in the $x$ and $y$ directions and two estimates for the displacements in the $z$ direction.

In order to analyze the displacement in planes oriented in the $x z$ direction, we took the $x z$ crosssections of our dataset for a range of $y$ values containing the TM. For each value of $y$ within this range, this gave us an $x z$ image for each recorded phase $\varphi$ of the stimulation signal. An $80 \times 10$ grid of voxels was created in the $x z$ plane. The displacement of each voxel relative to its initial location was estimated for each recorded value of $\varphi$, in both the $x$ and the $z$ direction.

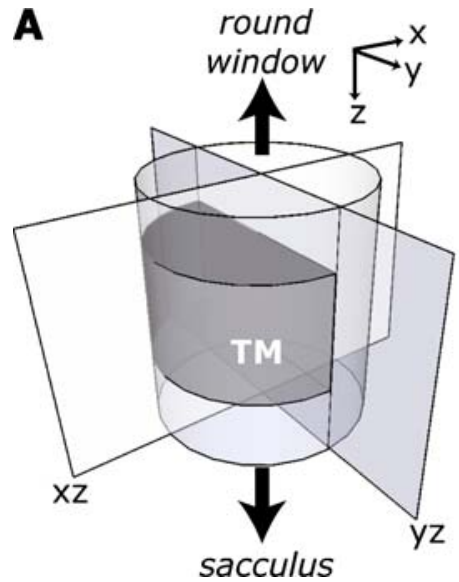

FIG. 3. A A schematic view of the $\mathrm{BP}^{\prime}$ s lumen and $\mathrm{TM}$; oriented as it is placed under the microscope. The viewing direction is $z$; from the round window to the sacculus. One $x z$ plane and one $y z$ plane, used in image analysis, are indicated in the figure. The half-moon shaped area indicates the TM. B a schematic view of an $x z$ cross-section. Hair bundles are drawn on the left, with the gray rectangle representing the TM covering them. The dashed rectangle indicates the TM, as it is expected to be displaced by acoustic stimulation. The double-headed arrow indicates the expected direction of the TM motion. C Definition
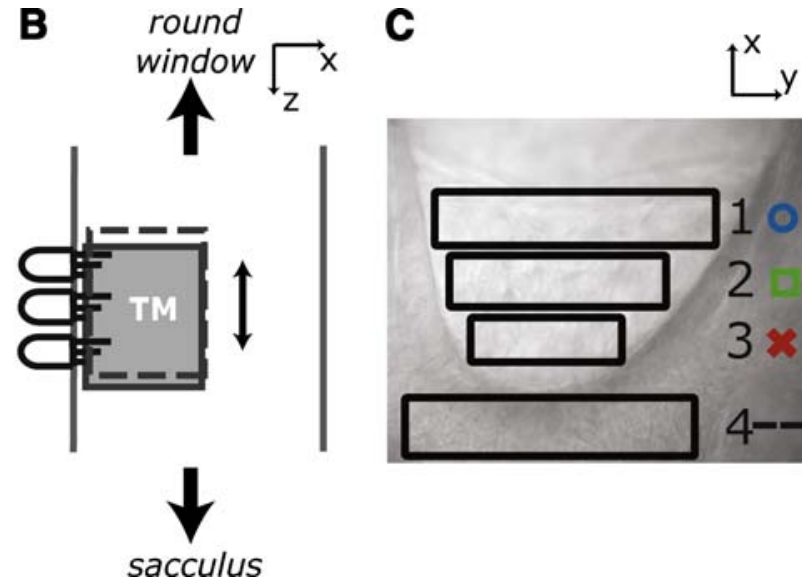

of the zones for the region-of-interest analysis. Zone 1 near the TM edge; zone 2 TM center; zone 3 near the hair cells; zone 4 in the lumen boundary/nerve fibers. The symbols to the right of the zone numbers correspond to those in Figure 6. These drawings assume perfect alignment of the TM displacement with the viewing direction. Our measurements indicate significant components of TM motion exist in both the $z$ direction and the $x$ direction, indicating that the epithelium was probably tilted by about $30^{\circ}$. 
The obtained displacements were then averaged in the $z$ direction. Next, a sinusoidal function in $\varphi$ was fitted to the averaged data. This resulted in 80 fitted amplitude and phase values for both the $x$ and the $z$ direction and for each value of $y$ within the range.

These fitted values were averaged in the $y$ direction, in order to smooth the results and create the same resolution in the $x$ and the $y$ directions. The resulting data gave an overview of the $x$ and the $z$ displacement amplitudes and phases, projected onto an xy plane.

The analysis was performed for each measurement frequency. For the estimation of the displacements in the $y$ direction and the second estimate in the $z$ direction, the procedure was repeated for planes oriented in the $y z$ direction of the original dataset. Here, a $60 \times 10$ grid of voxels in the $y z$ plane was used, reflecting the smaller size of the image in the $y$ direction.

The resulting data were read into Mathematica (version 5.2, Wolfram Research, Inc., Champaign, IL, USA) running on an Apple PowerMac computer. The amplitude data was linearly corrected for the variation in the stimulus amplitude; it was further smoothed using a running average over a $7 \times 7$ square of voxels.

ROI analysis For a more quantitative analysis of the data we defined four zones (Fig. 3C): (1) at the edge of the TM, (2) at the center of the TM, (3) at the hair-bundle-to-TM connection, and (4) outside the lumen in the surrounding tissue, generally in the nerve fibers.

Within each zone, we chose several ROIs with sufficient contrast to allow for a 3D displacement analysis. The number of ROIs was at least two and at most five per zone. The displacement data were then read into Matlab (version 7.5, The MathWorks, Natick, MA, USA) running on an Apple PowerMac computer. Displacements were averaged across the ROIs within each zone. For each direction, the data were fitted with a sinusoidal function in $\varphi$. This resulted in an estimate of the displacement amplitude and phase for each of the three spatial directions and for each zone.

\section{RESULTS}

\section{Cross-sectional views}

Figure 4 displays the displacement amplitudes for an individual preparation as color-coded density plots. In the plots, the analyzed image is partially displayed; only the area containing the TM and its direct surroundings are shown.

The amplitude of the displacement and the displaced area varied with frequency. The peak responses occurred at $2.0 \mathrm{kHz}$ for all three spatial directions, and at $0.5,1.0,3.0 \mathrm{kHz}$ the responses did not clearly exceed those of the lumen boundary. The highest peak- response amplitudes were between -10 and $-15 \mathrm{~dB}$, and in the $z$ direction. They occurred over a rather extensive area of the TM, including the zone near the hair-bundle attachments. This peak amplitude of the TM was approximately $15 \mathrm{~dB}$ larger than the stimulus amplitude at the operculum.

The overall response amplitude was lower in the $x$ and $y$ directions. Here, the peak was at approximately $-15 \mathrm{~dB}$. Also in these directions the amplitude distribution of the response was clearer: the peak responses occurred near the connections between the stereovilli and the TM. The shape and location of the TM became apparent from the amplitude graphs. The edge of the TM appeared to be displaced very little, especially near both connections to the lumen boundary.

The measured displacement of the lumen boundary was relatively high in the $\mathrm{z}$ direction (up to $-18 \mathrm{~dB}$ ). In the $x$ and $y$ directions the response of the lumen boundary was as low as $-30 \mathrm{~dB}$ or lower. The difference between the in-plane directions $(x y)$ and the $z$ direction could be ascribed to the difference in resolution. In order to evaluate the noise in our results, we conducted and analyzed measurements without any displacement of the operculum. The resulting amplitude overviews (not displayed) were similar to the response overviews at $500 \mathrm{~Hz}$.

In Figure 5, an overview is shown of the phase of the $x$ direction response for the specimen used for Figure 4. Arrows indicating the phase were superimposed onto an image of the BP from this specimen. The phase of the TM relative to the stimulation signal changed as a function of frequency, while the phase of the surrounding tissue remained constant across frequencies. The phase was approximately constant across the TM for each frequency, indicating that there were no frequency-dependent spatial modes of vibration within the BP's TM, or traveling waves in the $x$ direction. Phase overviews in the $y$ and $z$ directions are not shown. They gave the same general image of in-phase motion across the entire membrane and a similar frequency dependence of the phase.

\section{ROI analysis}

The ROI analysis provided a more quantitative description of the TM response in the three spatial directions. Figure 6 displays the amplitude and phase response for the specimen of Figures 4 and 5 .

In all three directions, all zones within the TM had a frequency-dependent response amplitude. The peak response was measured at $2.0 \mathrm{kHz}$. For all directions and most frequencies, the response amplitude was highest at the hair bundles (zone 3 ). The edge of the TM (zone 1) had the lowest amplitude response of the zones within the TM, while the lumen boundary 


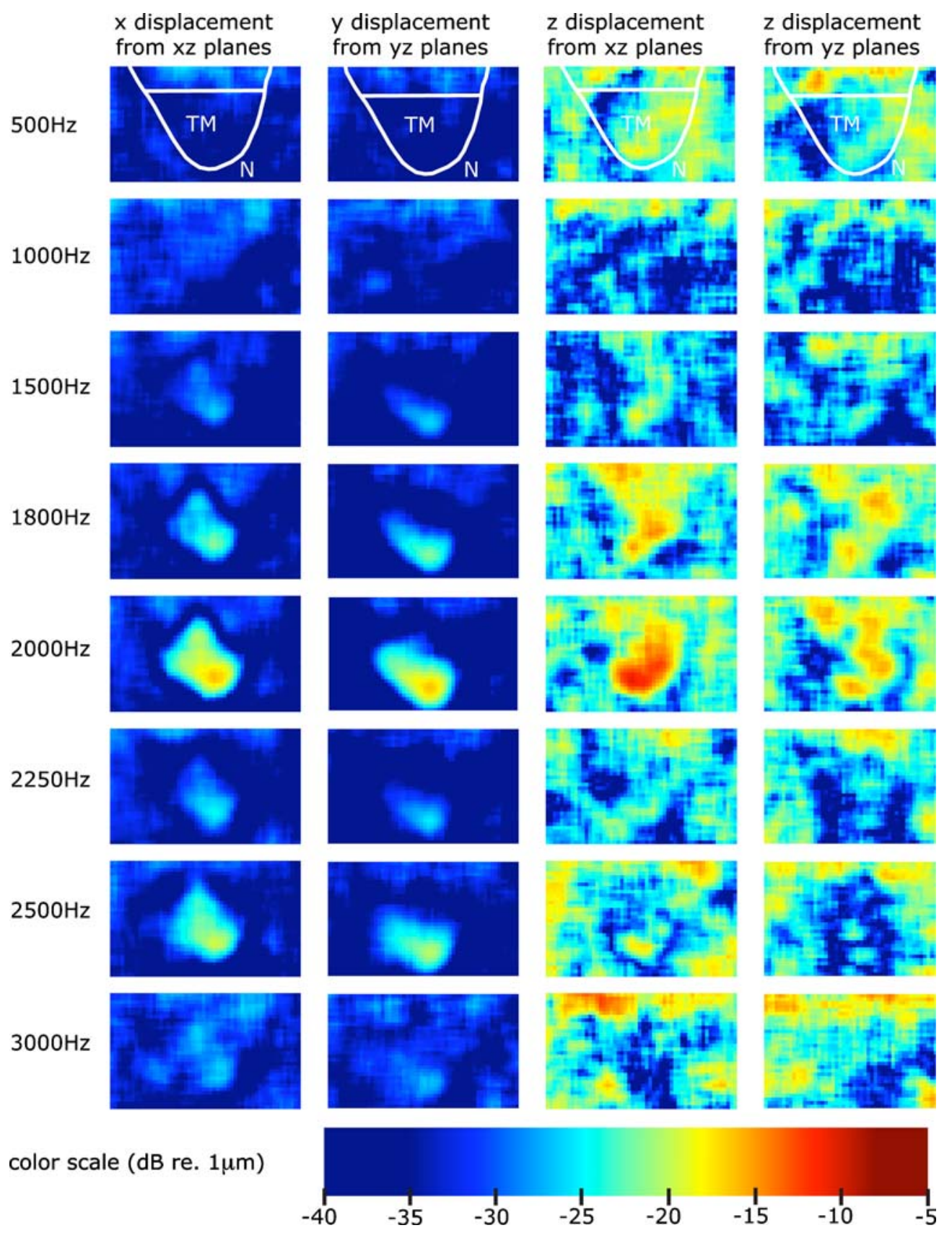

FIG. 4. Overview of amplitude responses in three directions for various measurement frequencies projected onto the $x y$ plane. The first and second columns indicate the displacement in the $x$ and $y$ direction, respectively, the third and fourths columns give both estimates of the $z$ displacement; the third column as calculated from

the $x z$ cross-sections, the fourth as calculated from the $y z$ crosssections. The stimulus amplitude was $0.05 \mu \mathrm{m}(-26 \mathrm{~dB}$ re. $1 \mu \mathrm{m})$ at the operculum. A sketched indication of the lumen boundary and of the location of the TM was drawn into the top row of graphs. Labels: $\mathrm{N}$ nerve fibers, $T M$ tectorial membrane.

(zone 4) had the overall lowest displacement, as was to be expected.

The response in the $z$ direction showed smaller differences between zones within the TM than the $x$ and $y$ responses. At the peak-response frequency $(2.0 \mathrm{kHz})$, the amplitude was approximately $-10 \mathrm{~dB}$ for all three zones within the TM. The lumenboundary-zone response (zone 4) was relatively constant across frequencies.
The peak responses in the $x$ and $y$ directions were about $3 \mathrm{~dB}$ lower than in the $z$ direction. Also the differences between the three zones within the TM were larger in the directions in the image plane. In the $x$ direction, the response of the TM's edge (zone 1) was well below the response of the rest of the membrane: at the peak response the difference between the TM's edge (zone 1) and the hair-bundle zone (zone 3) was $25 \mathrm{~dB}$, and it was at least $10 \mathrm{~dB}$ for each frequency. The 


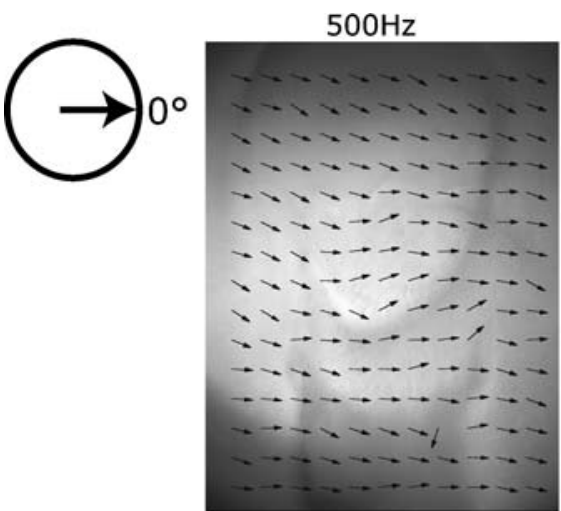

$2000 \mathrm{~Hz}$

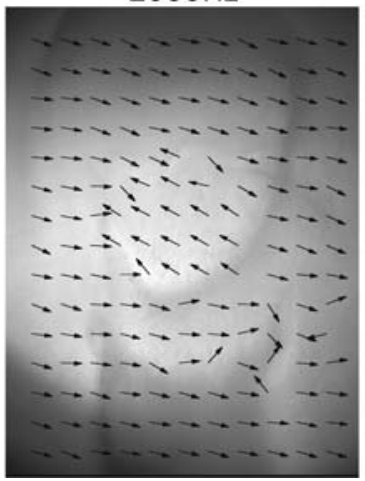

FIG. 5. The phase response for selected frequencies superimposed on a photo of the TM. The arrows indicate the phase of the displacement relative to the stimulus signal; phase $0^{\circ}$ is indicated

response of the center of the membrane (zone 2) was 2$5 \mathrm{~dB}$ below the response of the hair-bundle zone (zone 3), except for the response at $500 \mathrm{~Hz}$. The response of the lumen boundary (zone 4 ) was $10-15 \mathrm{~dB}$ below the TM-edge response (zone 1).

In the $y$ direction the response difference between the center (zone 2) and the hair-bundle zone (zone 3) was larger than in the $x$ direction $(3-10 \mathrm{~dB})$. Also, the response of the lumen boundary (zone 4) was similar to that of the TM-edge response (zone 1) in this direction.

Baseline measurements in six preparations gave us an indication of the measurement noise. We recorded response in absence of any stimulus. For in-plane directions these measurements produced in-plane response of $-54 \mathrm{~dB}$ averaged over four zones and eight measurements. The $z$ direction average baseline response was $-38 \mathrm{~dB}$ for the same dataset. The response of the lumen boundary in the $z$ direction is not significantly larger than the baseline response; the same can be said for the $x$ direction response at most frequencies.

The phase data, given in the bottom row of Figure 6 , confirmed the results in the overview of Figure 5. All three zones in the TM moved largely in phase for all three directions. The phase accumulated over at least $270^{\circ}$. Only in the $x$ direction, there was a limited phase difference between zones.
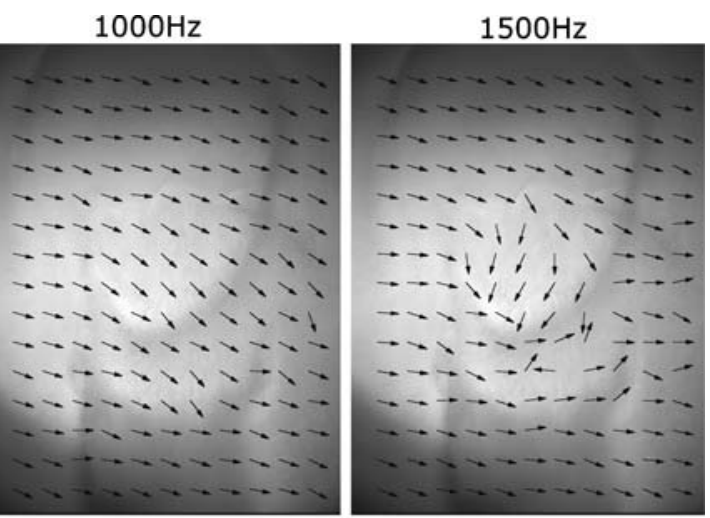

$2500 \mathrm{~Hz}$
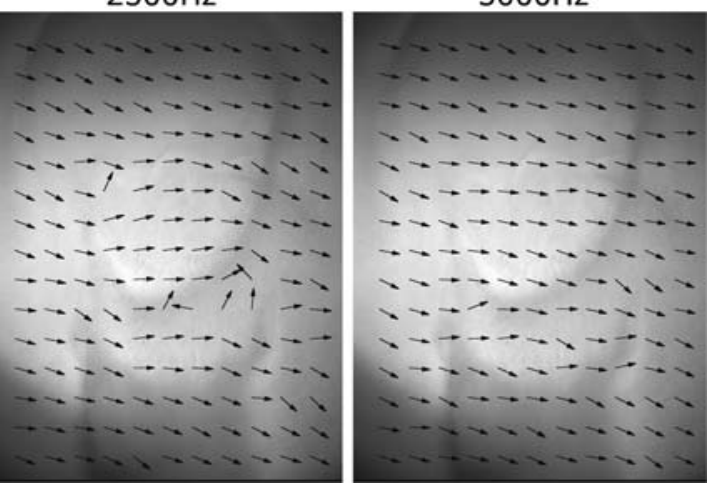

by an arrow to the right. The TM displays an increasing phase lag with increasing frequency. The phase data displayed are for the $x$ direction. The $y$ and $z$ directions give similar results.

In Figure 7, we combined the amplitude responses of six preparations from five animals for the hairbundle zone (zone 3). Between preparations, the peak-response frequency varied from 1.7 to $2.5 \mathrm{kHz}$. The response universally dropped at $3.0 \mathrm{kHz}$. In one specimen, we did not measure at frequencies higher than $2.0 \mathrm{kHz}$. The response data for this specimen were drawn in Figure 7 and showed a pattern consistent with the other preparations up to that frequency. Since this dataset did not cover the full frequency range, it was not taken into account for the quantitative data from here on.

In the $x$ and $z$ directions, the amplitudes for the different preparations showed a tuned response similar to the responses of the hair-bundle zone in Figure 6. Peak responses occurred between 2.0 and $2.5 \mathrm{kHz}$ and ranged from -27 to $-11 \mathrm{~dB}$ for the $x$ direction. The median value was $-15 \mathrm{~dB}$. The average dynamic range, as defined by the difference between the maximum and the minimum response within a preparation, was $27 \mathrm{~dB}( \pm 3 \mathrm{~dB}$, standard deviation). In the $z$ direction, peak-response frequencies were between 1.8 and $2.5 \mathrm{kHz}$. The peak amplitudes ranged from $-17 \mathrm{~dB}$ to $-10 \mathrm{~dB}$, with a median value of $-13 \mathrm{~dB}$. The dynamic range was lower than in the $x$ direction; on average $22 \mathrm{~dB}( \pm 5 \mathrm{~dB})$. The dynamic range in the $z$ direction may have been limited by the 


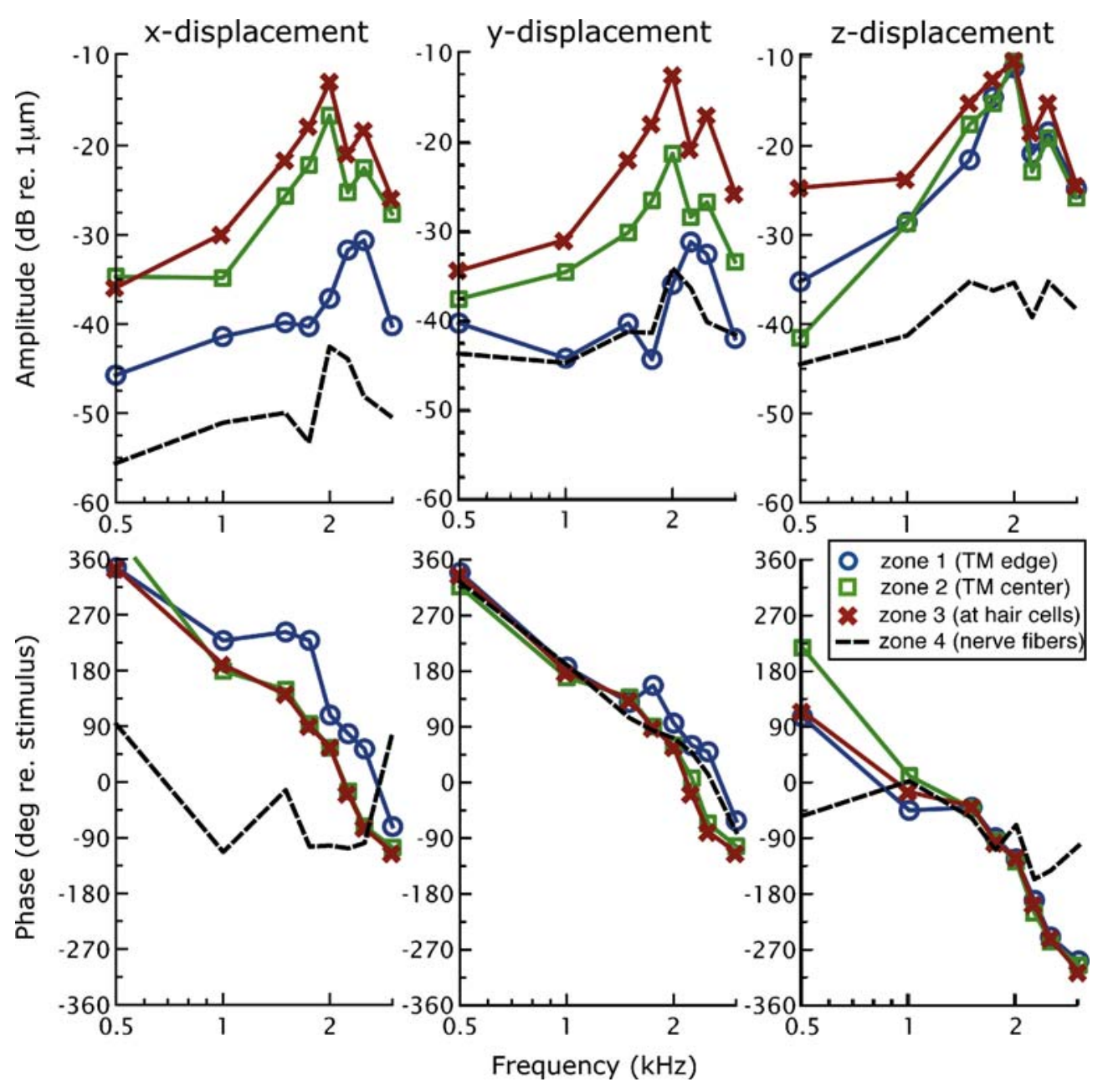

FIG. 6. The TM response in three directions to an operculum displacement with an amplitude of $0.05 \mu \mathrm{m}$. The top row gives the amplitude response; the bottom row the phase. Directions are indicated at the top of the figure. The columns represent the displace- ments in the $x, y$, and $z$ direction, respectively. The blue circles represent the TM's edge (zone 1), the green squares the center (zone 2 ), the red crosses the zone near the hair bundles (zone 3), and the dashed line the lumen boundary (zone 4); see Figure 3C. accuracy of our measurement, as the minimum of the across-preparation average corresponded to the baseline value. In the $y$ direction, the overall picture was less pronounced. While the maximum response occurred in the same frequency range $(1.7-2.5 \mathrm{kHz})$, it was lower; ranging from -36 to $-15 \mathrm{~dB}$, with a median of $-28 \mathrm{~dB}$.

From the tuning diagrams in Figure 7 , the peakresponse frequency was calculated to be $2.2 \mathrm{kHz}$ $( \pm 0.3 \mathrm{kHz}$, standard deviation), averaged across all three directions. The tuning sharpness in terms of $\mathrm{Q}_{10 d B}$ was determined for each direction and each specimen. On average, $\mathrm{Q}_{10 d B}$ was 2.4 ( \pm 1.0 , standard deviation), when averaged across all three directions. The average $Q_{10 d B}$ value in the $z$ direction was 2.0 $( \pm 0.8)$. The latter value is more indicative of the actual tuning of the TM, since the $z$ direction aligns most closely with the hair-cell orientation. In the $y$ direction, the value of the calculated tuning sharpness is debatable, since the tuned response is not obvious from the amplitude characteristics.

\section{DISCUSSION}

From the data presented in the "Results" section, the general mode of motion of the BP's TM under acoustic stimulation became apparent. The displacement of the membrane was highest near the attachment to the hair bundles and lowest near the edge that spans across the lumen. The phase was approximately constant across the TM. Overall the motion can therefore be likened to that of a two-dimensional pendulum, hinged at the TM's edge; or a semicircular cat shutter. We did not obtain any evidence of largescale distortion of the TM in our recordings. The fact that the maximum displacement occurred near the 

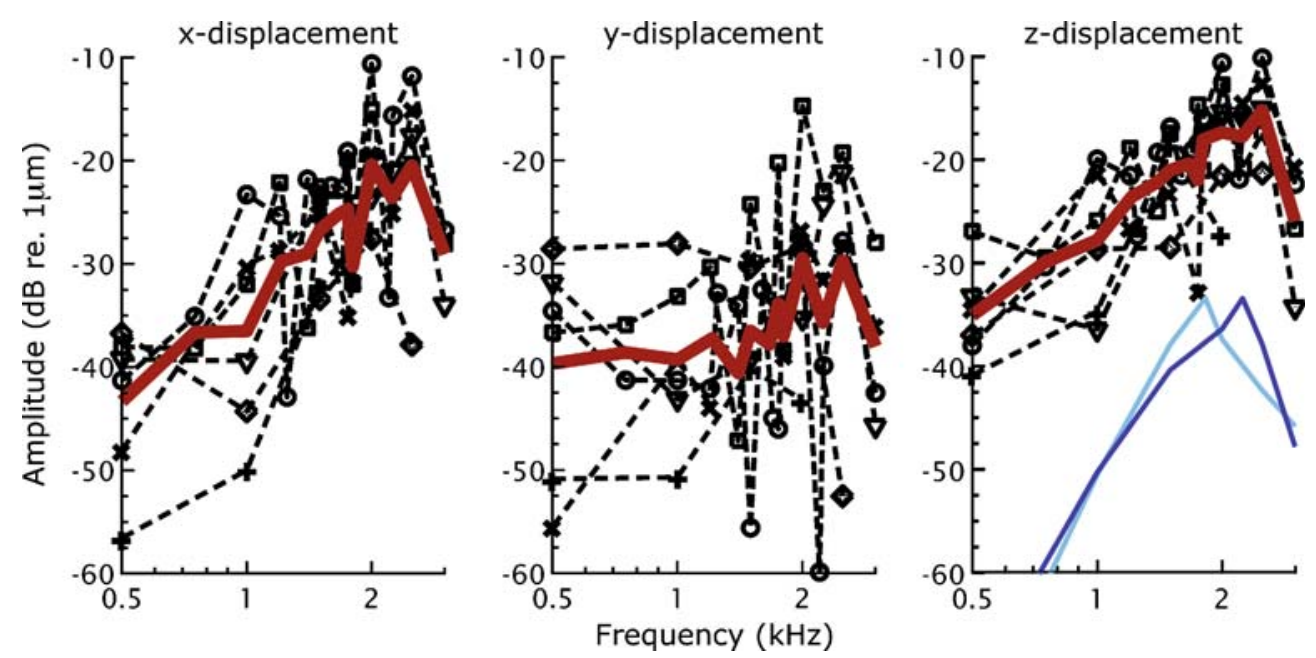

FIG. 7. Amplitude data in three directions for six preparations. The amplitudes displayed are the averaged values of the regions of interest near the hair-bundles attachments (zone 3). Data were scaled linearly to the same stimulus amplitude across preparations; this amplitude was $0.04 \mu \mathrm{m}$. The dashed lines indicate the individual preparations.

hair cells is in line with the TM's function in the detection of sound. The lower response amplitudes at the TM's edge presumably resulted from the membrane being thicker in this area and its firm attachments to the lumen boundary (Van Bergeijk 1957; Frishkopf and Flock 1974).

These experiments were conducted in isolated inner-ear preparations. Data collections in the first preparation typically started $1 \mathrm{~h}$ post mortem and were continued for a maximum of $4 \mathrm{~h}$. In the BP, distortion product otoacoustic emissions (DPOAEs) disappear on average approximately half an hour after the blood supply is stopped (Van Dijk et al. 2003). Therefore, our experiments were conducted in a post mortem time frame for which active hair-cell involvement had presumably ceased. Over the duration of the experiments, we did not observe any degradation of the response, nor did we observe visible changes in the structure of hair cells or the TM. Additionally, scanning electron-microscopy photographs were taken of some of the preparations (results not shown); these preparations did not show more damage to the hair cells than previously unused preparations. As a control experiment we deliberately ruptured the contact membrane in a preparation. Over the typical duration of an experiment, the mixing of perilymph and endolymph fluids led to observable swelling in the hair-cell bodies in this damaged preparation; a condition that clearly differed from the typical conditions during the experiments. These combined observations suggest that the structural properties of the epithelium were maintained in their normal state, although any active processes had presumably ceased.
The thick, red drawn line indicates the $d B$ values averaged across preparations. The blue lines indicate two neural-tuning curves based on data from Ronken (1990, Fig. 1). These were flipped and shifted in the vertical direction to facilitate comparison.

The detected peak amplitude of the TM exceeded the input amplitude at the operculum by about $15 \mathrm{~dB}$. Apparently the structure of the frog's inner ear labyrinths results in an acoustic lever that enhances the displacement amplitude at the auditory receptor. In mammals, the gain between basilar membrane and stapes motion depends highly on the cochlea's active amplification process. At the best frequency and at low sound pressure levels, basilar membrane/stapes gains as high as $70 \mathrm{~dB}$ have been reported (Robles and Ruggero 2001). Due to the amplifier's nonlinear compression, the gain is reduced substantially at high sound pressure levels and is also reduced post mortem, when the cochlear response is basically linear. For example, one study showed a basilar membrane/stapes gain at the best frequency of approximately $50 \mathrm{~dB}$ when the stimulus level was $20 \mathrm{~dB}$ SPL, dropping to a gain of approximately $20 \mathrm{~dB}$ when the stimulus level was $90 \mathrm{~dB}$ SPL. Post mortem the gain was also about $20 \mathrm{~dB}$ at the best frequency. Somewhat below the best frequency the gains were substantially lower, much less level-dependent and did not change much post mortem, with values of about 20-30 dB (Ren and Nuttall 2001). The post-mortem gain value of about $20 \mathrm{~dB}$ from the stapes to $\mathrm{BM}$ in the mammalian cochlea is similar to what we see in the gain between the operculum (oval window) and the TM of the frog.

The response of the TM was frequency-selective, with a peak response at $2.2 \mathrm{kHz}( \pm 0.3 \mathrm{kHz}$, standard deviation). This is in correspondence with the expected neural peak response in the $\mathrm{BP}$ of $R$. pipiens pipiens (Ronken 1991; Meenderink et al. 2005b), be it at the high end of the expected range. The phase of 
the TM motion accumulated over at least $270^{\circ}$, indicating that the response may have been shaped by a filter of order three or higher.

The tuned response in the $x$ and $y$ directions was presumably the result of an imperfect alignment between the line of sight ( $z$ direction) and the haircell orientation. An angle between the two would lead to components in the $x y$ plane for motion in the direction of the hair-bundle orientation. We calculated the misalignment under the assumption that the epithelium was flat and the TM moved along it. Based on the averaged peak-amplitude data, the angle between perfect and actual alignment would be $30^{\circ}$ in the $x z$ plane and $10^{\circ}$ in the $y z$ plane. The $x$ direction angle seems reasonable assuming that the location of the contact membrane in our preparations is comparable to that in the bullfrog (see Fig. 1C; Frishkopf and Flock 1974, Fig. 2a). The fact that the $y$ direction angle was smaller may have been the result of our use of the symmetry of the TM for the alignment of the preparation. Therefore, we do not discard the notion that the TM motion is strictly along the sensory epithelium. Such a displacement would be consistent with in the $180^{\circ}$ phase difference between the $x$ and $z$ responses of the TM (Fig. 6).

The $\mathrm{Q}_{10 d B}$ determined from the tuning of the TM near the hair-bundle attachments gave an average value of $2.0 \quad( \pm 0.8$, standard deviation $)$ in the $z$ direction. This measure of tuning sharpness is of the same order of magnitude as the values reported for the nerve fiber tuning curves in $R$. pipiens pipiens (Ronken 1991). The $\mathrm{Q}_{10 d B}$ values could not be expected to correspond exactly, due to the difference in measurement parameters: We measured the TM motion in response to a constant stimulus amplitude, while nerve-fiber tuning is measured as the required stimulus to achieve a predefined spike-rate increase. Iso-input, neural tuning curves are known for the BP of $R$. esculenta that has best frequencies that are similar to the peak-response frequencies we found. The $\mathrm{Q}_{10 d B}$ values determined from these responses range from 1.0 to 3.0 (Van Dijk et al. 1997, Fig. 9a, $f>1.0 \mathrm{kHz}$ ). This corresponds closely to the tuning we found in the TM response.

Finally, the tuning sharpness of the contact membrane in the bullfrog $\left(Q_{10 d B}=1.4 \pm 0.4\right.$, average \pm standard deviation) determined from Purgue and Narins (2000a, Fig. 6) is lower than that in our measurements of the TM's reponse in the leopard frog. Although the difference is not statistically significant, this suggests that the response of the contact membrane only partially reflects the frequency selectivity of the BP.

The tuned response of the BP's TM is presumably the result of a combination of various anatomical features. The large-scale fluid dynamics of the inner ear and the tuning of the contact membrane (Purgue and Narins 2000b) may provide initial filtering of the acoustic energy passing through the BP. The response of the TM may then be tuned further by its own mass and stiffness, and the stiffness of its connections to the lumen boundary, including the hair-bundle stiffness at the epithelium. From our current data, it is not possible to determine the relative contributions of the individual components.

In conclusion, the response of the basilar papilla's tectorial membrane in the northern leopard frog is mechanically tuned to a frequency near $2.0 \mathrm{kHz}$, consistent with the characteristic frequencies of auditory neurons connecting to this organ. The phase response implied a filtering mechanism of at least the third order. Tuning sharpness was comparable to nerve-fiber tuning sharpness and the tuning sharpness of the contact membrane in a related species. Thus, the frog's basilar papilla is an auditory receptor without a basilar membrane, in which the frequency selectivity is based on the mechanical response of the tectorial membrane.

\section{ACKNOWLEDGEMENTS}

The authors would like to thank Dr. D.M. Freeman and Dr. A.J. Aranyosi (Research Laboratory of Electronics, Massachusetts Institute of Technology) for making their image analysis software available and the introduction to its usage. Also, we would like to thank three anonymous reviewers and the editors for the many useful comments. This study was supported by the Heinsius Houbolt Foundation and the Netherlands Organisation for Scientific Research and is part of our department's research program: Communication through Hearing and Speech. The results discussed in this paper were presented in part at The Tenth International Workshop on the Mechanics of Hearing (July 2008, Keele, UK).

\section{Open Access}

This article is distributed under the terms of the Creative Commons Attribution Noncommercial License which permits any noncommercial use, distribution, and reproduction in any medium, provided the original author(s) and source are credited.

\section{REFERENCES}

Davis C, Freeman D. Using a light microscope to measure motions with nanometer accuracy. OptEng. 37:1299-1304, 1988.

Evans EF. The sharpening of cochlear frequency selectivity in the normal and abnormal cochlea. Audiology 14(5-6):419-442, 1975.

FrishKOPF LS, FLOCK A. Ultrastructure of the basilar papilla, an auditory organ in the bullfrog. Acta Otolaryngol 77(3):176-184, 1974.

Horn BK, Weldon E, JR. Direct methods for recovering motion. IJCV 2:51-76, 1988. 
LEwIS ER. Surface morphology of the bullfrog amphibian papilla. Brain Behav. Evol. 13(2-3):196-215, 1976.

LEwIS ER, NARINS PM. The acoustic periphery of amphibians; anatomy and physiology. In: Fay RR, Popper AN (eds) Comparative Hearing: Fish and Amphibians. New York, Springer, pp. 101-154, 1999.

Lewis ER, Leverenz EL, Kovama H. The tonotopic organization of the bullfrog amphibian papilla, an auditory organ lacking a basilar membrane. J. Comp. Physiol. 145:437-445, 1982.

MeEnderink SWF, Narins PM, VAn Dijk P. Detailed f1, f2 area study of distortion product otoacoustic emissions in the frog. J. Assoc. Res. Otolaryngol. 6(1):37-47, 2005a.

MeEnderink SWF, VAN DiJK P, NARINS PM. Comparison between distortion product otoacoustic emissions and nerve fiber responses from the basilar papilla of the frog. J. Acoust. Soc. Am. 117(5):3165-73, 2005b.

Purgue AP, Narins PM. Mechanics of the inner ear of the bullfrog (Rana catesbeiana): the contact membranes and the periotic canal. J. Comp. Physiol. A 186:481-488, 2000a.

Purgue AP, Narins PM. A model for energy flow in the inner ear of the bullfrog (Rana catesbeiana). J. Comp. Physiol. A 186:489495, $2000 \mathrm{~b}$.

Ren T, NutTall A. Basilar membrane vibration in the basal turn of the sensitive gerbil cochlea. Hear Res. 151:48-60, 2001.

Robins RG, Bauknight RS, Honrubia V. Anatomical distribution of efferent fibers in the 8th cranial nerve of the bullfrog (Rana catesbeiana). Acta Otolaryngol. 64(5):436-448, 1967.

Robles L, Ruggero MA. Mechanics of the mammalian cochlea. Physiol. Rev. 81(3):1305-1352, 2001.

RonKen DA. Basic properties of auditory-nerve responses from a 'simple' ear: the basilar papilla of the frog. Hear Res. 47:63-82, 1990.
Ronken DA. Spike discharge properties that are related to the characteristic frequency of single units in the frog auditory nerve. J. Acoust. Soc. Am. 90:2428-2440, 1991.

Schoffelen RLM, Segenhout JM, Van Dijk P. Motion of the tectorial membrane in the basilar papilla of the northern leopard frog, rana pipiens. In: Abstracts of the thirtieth annual midwinter research meeting, ARO, p 171, 2007.

Schoffelen RLM, Segenhout JM, van DiJK P. Mechanics of the exceptional anuran ear. J. Comp. Physiol. A 194(5):417-428, 2008.

Simmons D, Meenderink S, Vassilakis P. Anatomy, physiology, and function of auditory end-organs in the frog inner ear. In: Narins PM, Feng AS, Fay RR, Popper AN (eds) Hearing and Sound Communication in Amphibians. New York, Springer, pp. 184-220, 2007.

VAN BERGEIJK WA. Observations on models of the basilar papilla of the frog's ear. J. Acoust. Soc. Am. 29(11):1159-1162, 1957.

VAn DijK P, Meenderink S. Distortion product otoacoustic emissions in the amphibian ear. In: Nuttall AL, Ren T, Gillespie P, Grosh K, de Boer E (eds) Auditory Mechanisms, Processes and Models. Singapore, World Scientific, pp. 332-338, 2006.

VAN DIJK P, NARINS PM, WANG J. Spontaneous otoacoustic emissions in seven frog species. Hear Res. 101:102-112, 1996.

VAn Dijk P, Wit HP, Segenhout JM. Dissecting the frog inner ear with gaussian noise, i, application of high-order wiener-kernel analysis. Hear Res. 114(1-2):229-242, 1997.

VAN DIJK P, NARINS PM, Mason MJ. Physiological vulnerability of distortion product otoacoustic emissions from the amphibian ear. J. Acoust. Soc. Am. 114:2044-2048, 2003.

Wever EG. The Amphibian Ear. Princeton, Princeton University Press, 1985. 